\title{
Geometric Structures and Magnetic Interactions in Small Chromium Oxide
}

\section{Clusters}

Le Nhan Pham,,$+\neq$ Pieterjan Claes, $"$ Peter Lievens, "Ling Jiang,, , $\|$ Torsten Wende, ${ }^{\S}$ Knut R. Asmis, ${ }^{\S}$ Minh Tho Nguyen, ${ }^{*, \dagger}$ and Ewald Janssens, ${ }^{*}$

$\dagger$ Department of Chemistry, KU Leuven, Celestijnenlaan 200F, B-3001 Leuven, Belgium $\ddagger$ Department of Chemistry, The University of Dalat, 670000 Dalat, Vietnam \Laboratory of Solid State Physics and Magnetism, KU Leuven, Celestijnenlaan 200D, B-3001 Leuven, Belgium

$\S$ Wilhelm-Ostwald-Institute for Physical and Theoretical Chemistry, University of Leipzig, Linnestrasse 2, D-04103 Leipzig, Germany

||Current address: State Key Laboratory of Molecular Reaction Dynamics, Collaborative Innovation Center of Chemistry for Energy and Materials, Dalian Institute of Chemical Physics, Chinese Academy of Sciences, Dalian 116023, Liaoning, P. R. China

E-mail: minh.nguyen@kuleuven.be; ewald.janssens@kuleuven.be Phone: +32 163272 07. Fax: +32 16327983 


\begin{abstract}
The physical and chemical properties of transition metal oxide particles result from the subtle interplay between atomic ordering and electronic structure, the latter being determined by a complex interaction between the partially filled $d$ subshell of the transition metal atoms and the oxygen $2 p$ orbitals. In this article, the geometric ground state structures of several experimentally synthesized cationic chromium oxide clusters $\mathrm{Cr}_{\mathrm{m}} \mathrm{O}_{\mathrm{n}}{ }^{+}(\mathrm{m}=2,3,4 ; \mathrm{n} \leq \mathrm{m})$ are characterized through infrared photodissociation spectroscopy on cluster-rare gas atom complexes in combination with quantum chemical calculations. Computational analysis of the electronic and magnetic properties of the identified isomers demonstrated that the magnetic configuration of the clusters varies with the size and oxidation state. Superexchange interaction causes ferromagnetic coupling in $\mathrm{Cr}_{2} \mathrm{O}_{2}{ }^{+}$and $\mathrm{Cr}_{3} \mathrm{O}_{3}{ }^{+}$, while $3 d-3 d$ bonding-like interaction between two chromium atoms underlies ferrimagnetic behavior in $\mathrm{Cr}_{3} \mathrm{O}^{+}, \mathrm{Cr}_{3} \mathrm{O}_{2}{ }^{+}$, and $\mathrm{Cr}_{4} \mathrm{O}_{4}{ }^{+}$. The highest possible total magnetic moments are obtained in suboxides that have $\mathrm{Cr}-\mathrm{O}-\mathrm{Cr}$ bridges with a unique oxygen atom between each pair of $\mathrm{Cr}$ atoms. The addition of more oxygen atoms enhances the delocalization of the Cr $3 d$ electrons and reduces the magnetic moment.
\end{abstract}

\title{
Introduction
}

Bulk chromium is an antiferromagnetic material at room temperature (its Néel temperature is about $308 \mathrm{~K}$ ) and behaves paramagnetically at higher temperatures. ${ }^{1}$ The chromium atom has, in the ground state, six unpaired electrons in its $3 d$ and $4 s$ orbitals. In the smallest possible cluster of chromium atoms, the $\mathrm{Cr}_{2}$ dimer, the total magnetic moment is zero as a result of anti-ferromagnetic coupling of the local moments. ${ }^{2-4}$ Interestingly, the $\mathrm{Cr}-\mathrm{Cr}$ bond length is significantly increased and the local moments at the two chromium sites couple ferromagnetically if one electron is removed resulting in a high-spin ground state $\left({ }^{12} \Sigma\right)$ for $\mathrm{Cr}_{2}{ }^{+} \cdot{ }^{4}$ Triatomic and other small odd sized chromium clusters are subject 
to antiferromagnetic frustration. ${ }^{5,6}$ In a recent theoretical study, an energetic competition between low and high spin states $(\mathrm{S}=1$ and $\mathrm{S}=6)$ was found in $\mathrm{Cr}_{4}$ as a result of the highly correlated electron behavior. ${ }^{7}$

Magnetic interactions in pure chromium clusters can be altered by addition of oxygen atoms. Superexchange through bridging oxygen atoms induces a ferromagnetic coupling between the local moments on the two $\mathrm{Cr}$ atoms in $\mathrm{Cr}_{2} \mathrm{O}_{\mathrm{n}}{ }^{-/ 0}(\mathrm{n}=1-3) .^{8,9}$ Further addition of oxygen reduces, and even quenches, total magnetic moments of the dichromium clusters $\left(\mathrm{Cr}_{2} \mathrm{O}_{\mathrm{n}}, \mathrm{n}=4-14\right) .{ }^{10,11}$ A similar observation was made for larger clusters; the local magnetic moments of chromium atoms are quenched in clusters with a high oxygen concentration, such as $\mathrm{Cr}_{4} \mathrm{O}_{10}$ and $\left(\mathrm{CrO}_{3}\right)_{\mathrm{n}}(\mathrm{n}=1-5) .{ }^{12,13}$ Such quenching happens because all chromium valence electrons are involved in chemical bonds with oxygen atoms. In suboxide clusters, such as $\mathrm{Cr}_{3} \mathrm{O}_{\mathrm{n}}^{+/ 0}(\mathrm{n}=0-3)$, the magnetic interactions are controlled by the amount of oxygen. ${ }^{5}$ Besides the composition (chromium to oxygen atomic ratio), also the precise geometry of the cluster is important for the magnetic interaction. It was, for example, found that $\mathrm{Cr}_{\mathrm{n}} \mathrm{O}_{2}(\mathrm{n}=2-5)$ clusters of the same size but with a different geometric structure can have different magnetic properties and interactions. ${ }^{14,15}$ Similar findings were reported for iron oxide clusters. For example, different magnetic states were predicted as lowest energy configuration of the neutral $\mathrm{Fe}_{4} \mathrm{O}_{6}$, although these isomers have similar geometries ( $\mathrm{T}_{d}$ versus slightly distorted $\mathrm{T}_{d}$ symmetry), including a ferromagnetic state with a magnetic moment of $20 \mu_{B},{ }^{16}$ a ferrimagnetic $10 \mu_{B}$ state, ${ }^{17}$ and most recently a singlet state $\left({ }^{1} \mathrm{~A}_{2}, \mathrm{C}_{2 v}\right) \cdot{ }^{18,19}$ Therefore it is important to identify the geometry of clusters produced in the experiment before assessing their magnetic ground state configurations. The cluster geometry can be accurately obtained from infrared photodissociation (IRPD) spectroscopy experiments on cluster-rare gas atom complexes. The detachment of the rare gas atoms is detected mass spectrometrically and signals resonant absorption of infrared photons. This spectroscopic technique, in combination with density functional theory (DFT) calculations, has been proven a powerful technique to identify the geometries of small metal oxide clus- 
ters. ${ }^{18,20-24}$

To fully understand the interplay between chemical compositions, geometric arrangements, and magnetic properties of small chromium oxide clusters, we synthesized a series of cationic $\mathrm{Cr}_{\mathrm{m}} \mathrm{O}_{\mathrm{n}}{ }^{+}(\mathrm{m}=2,3,4 ; \mathrm{n} \leq \mathrm{m})$ clusters and studied them by a combination of infrared spectroscopy and quantum chemical calculations. Several quantum chemical methods were employed to locate ground-state and low-lying structures of the synthesized clusters. The magnetic interactions between and/or among local sites were studied. The evolution of magnetic moments through chemical control is discussed.

\section{Experimental Methods}

The experiments were carried out using a molecular beam setup consisting of a cluster source, an ion guide, a mass filter, an ion trap, and a time-of-flight mass spectrometer. ${ }^{25}$ This setup was temporarily installed at the Free Electron Laser for Infrared eXperiments (FELIX) facility (The Netherlands). ${ }^{26}$ The chromium oxide clusters were prepared by a pulsed laser vaporization source operated at $10 \mathrm{~Hz} \cdot{ }^{20,27}$ The $2^{\text {nd }}$ harmonic of a Nd-YAG laser is focussed on a metallic $\mathrm{Cr}$ plate and creates a plasma, which is entrained in a pulse of $1 \% \mathrm{O}_{2}$ seeded in He carrier gas and expanded through a clustering channel. The chromium-oxide cluster beam is collimated and thermalized to room temperature by collisions with Ar atoms in a radio frequency $(\mathrm{RF})$ decapole ion guide and mass-selected by a quadrupole mass filter. Mass-selected cationic clusters are accumulated in a ring electrode ion trap. To allow for continuous ion loading, ion thermalization, and cluster-rare gas (He, $\mathrm{Ne}$ ) complex formation, the trap is continuously filled with a buffer gas of either pure $\mathrm{He}\left(\right.$ for $\mathrm{Cr}_{3} \mathrm{O}^{+}$and $\mathrm{Cr}_{3} \mathrm{O}_{2}{ }^{+}$) or a mixture of $0.125 \% \mathrm{Ne}$ in $\mathrm{He}\left(\right.$ for $\mathrm{Cr}_{2} \mathrm{O}_{2}{ }^{+}, \mathrm{Cr}_{3} \mathrm{O}_{3}{ }^{+}$, and $\mathrm{Cr}_{4} \mathrm{O}_{4}{ }^{+}$) at ion trap temperatures in the range of 20-35 K. After filling the trap for $98 \mathrm{~ms}$, all ions are extracted and focused both temporally and spatially into the center of the extraction region of an orthogonally mounted linear time-of-flight (TOF) mass spectrometer. Here, the ion packet can be irradiated with 
the IR laser pulse. The absorption of a few and often only a single IR photon(s) by the weakly bound cluster-rare gas ionic complex is sufficient for vibrational predissociation and loss of the rare gas messenger. Scanning the wavelength of the excitation laser light provides the IRPD spectra, which are obtained in the difference mode of operation (laser on-laser off) and recorded by monitoring all ion intensities simultaneously. The infrared free electron laser FELIX is operated in the $400-750 \mathrm{~cm}^{-1}$ spectral range at a repetition rate of $5 \mathrm{~Hz}$, a bandwidth of $0.2 \%$ root mean square of the central wavelength and an average pulse energy of $10 \mathrm{~mJ}$.

\section{Computational Methods}

The CALYPSO ${ }^{28}$ tool was used to generate large numbers of initial structures at relatively low computational levels (B3LYP/3-21G and BP86/3-21G). The generated low-lying energetic structures were subsequently reoptimized with the larger cc-pVTZ basis set. ${ }^{29,30}$ Different spin multiplicities were tested for all structural isomers. Various density functionals (B3LYP, ${ }^{31-33}$ B3P86, ${ }^{33,34}$ B3PW91, ${ }^{33}$ BP86, ${ }^{32,34}$ TPSS, ${ }^{35}$ TPSSh, and M06L ${ }^{36}$ ) have been tested to assure reliability of the obtained results. In addition, for $\mathrm{Cr}_{2} \mathrm{O}_{2}^{+}$the restricted active space followed by second-order perturbation treatment (RASSCF/RASPT2) and coupled cluster (CCSD(T)-F12) methods have been used as benchmark for the DFT calculations. More details about these computational processes are provided in the Supporting Information (SI). Throughout the calculation process, the TPSS functional appears to be excellent in vibrational simulations of the obtained clusters in comparison to the other used functionals; therefore the simulated spectra from this functional are used as the basis for 4 out of 5 spectral assignments in this work. The B3P86 functional was used for $\mathrm{Cr}_{2} \mathrm{O}_{2}{ }^{+}$, since the IR spectrum of its ${ }^{8} \mathrm{~B}_{1 u}$ state could not be assessed at the TPSS level (see SI for details). DFT energies were corrected for zero point energies (ZPEs) at corresponding levels of theory, unless otherwise stated. The harmonic vibrational frequencies of the ground and first ex- 
cited states were analytically obtained after DFT optimizations. If not explicitly specified in the text, harmonic vibrational frequencies were calculated with the Gaussian 09 program. ${ }^{37}$ The empirical dispersion correction GD3 ${ }^{38}$ (for supported functionals) for optimizations and spectral simulations was taken into account.

\section{Results and discussion}

\section{Structural assignments}

Starting from the simplest synthesized cluster $\mathrm{Cr}_{2} \mathrm{O}_{2}{ }^{+}$, two clear bands around 610 and 720 $\mathrm{cm}^{-1}$ were observed from the experimental IRPD spectrum of the $\mathrm{Cr}_{2} \mathrm{O}_{2}{ }^{+} \cdot \mathrm{Ne}$ complex. The ground, first excited states and other low-lying ones of $\mathrm{Cr}_{2} \mathrm{O}_{2}{ }^{+}$are listed in Table $\mathrm{S} 1$ of the SI. At all considered levels of theory the ${ }^{8} \mathrm{~B}_{1 g}$ and ${ }^{8} \mathrm{~B}_{1 u}$ states are much lower in energy, so we just focus on these two states. Comparing the geometrical structures of the ${ }^{8} \mathrm{~B}_{1 g}$ ground and the ${ }^{8} \mathrm{~B}_{1 u}$ excited states, small differences are found. The $\mathrm{Cr}-\mathrm{O}$ bond is $0.01 \AA$ longer when $\mathrm{Cr}_{2} \mathrm{O}_{2}{ }^{+}$is stimulated from the ${ }^{8} \mathrm{~B}_{1 g}$ ground state to the ${ }^{8} \mathrm{~B}_{1 u}$ first excited state. The bonding angles $\widehat{O C r} O$ and $\widehat{C r O C} r$ also stretch and bend, respectively, 5 degrees in comparison to the ones of the ground state. In terms of electronic structures, these two states differentiate from each other in the occupation of electrons in $\mathrm{b}_{1 g}$ and $\mathrm{b}_{1 u}$ orbitals. The leading configurations of these different electronic states are given in Table S2 of the SI.

The difference in energy between the ground state ${ }^{8} \mathrm{~B}_{1 g}$ and first excited state ${ }^{8} \mathrm{~B}_{1 u}$ at the RASPT2 level is $0.38 \mathrm{eV}$. Assuming a Maxwell-Boltzmann statistics, occupancy of an excited state at $+0.38 \mathrm{eV}$ would be small. However, besides uncertainty on the calculated relative energy, also the attachment of messenger rare gases may affect the energy differences between isomers. Due to shortcomings of the implementation of the RASPT2 calculational technique, the effect of rare gas messenger atoms on the excited states of $\mathrm{Cr}_{2} \mathrm{O}_{2}{ }^{+}$cannot be examined precisely. Taking into account the computational uncertainty of RASPT2 and other quantum chemical methods, the differences in geometrical structures of the ground 
and first excited states are insignificant, and it is well possible that both the ground and first excited state are populated in the experiment. Similar observations have recently been made for $\mathrm{Cr}_{2} \mathrm{O}_{2}{ }^{-/ 0} \cdot{ }^{39}$

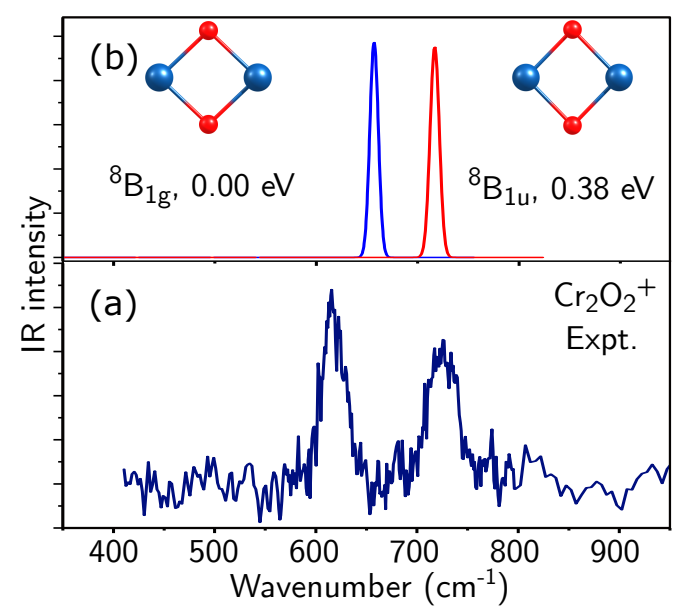

Figure 1: (a) Experimental IRPD spectra and (b) simulated harmonic IR spectra of $\mathrm{Cr}_{2} \mathrm{O}_{2}{ }^{+}$. The simulated spectrum of the ${ }^{8} \mathrm{~B}_{1 g}$ ground state is presented by the blue line, the spectrum of the ${ }^{8} \mathrm{~B}_{1 u}$ excited state by the red line. The geometries of the lowest-lying states are shown as insets.

Although the TPSS functional is used for simulating the vibrational spectra of most clusters studied in this work, the simulated IR spectrum of excited state ${ }^{8} \mathrm{~B}_{1 \mathrm{u}}$ could not be accessed at this level; therefore, the simulated spectra of these two states at the B3P86 level are used in Figure 1b. The simulated IR spectrum in Figure 1b is consistent with those obtained for the same isomer using other DFT functionals (TPSSH, B3PW91, B3P86, B3LYP, M06L, and BP86) (see Figure S9 of the SI). Note that the interaction of the cationic chromium oxide clusters with the rare gas messenger atoms ( $\mathrm{Ne}$ and $\mathrm{He}$ ) does not significantly affect the simulated IR spectra (see Figure S2 of the SI).

The simulated IR spectrum of the ground state $\left({ }^{8} \mathrm{~B}_{1 g}\right)$ shows a single peak at around 630 $\mathrm{cm}^{-1}$, which can only explain one of two experimental bands (Figure 1a). The first excited ${ }^{8} \mathrm{~B}_{1 u}$ state's simulated spectrum has a vibrational mode at around $700 \mathrm{~cm}^{-1}, 70 \mathrm{~cm}^{-1}$ above that of the ${ }^{8} \mathrm{~B}_{1 g}$ ground state (see Figure $1 \mathrm{~b}$ ). Their separation agrees reasonably well with the observed band separation of $90 \mathrm{~cm}^{-1}$ in the experimental spectrum. Therefore, both 
the ${ }^{8} \mathrm{~B}_{1 g}$ ground state and the ${ }^{8} \mathrm{~B}_{1 u}$ first excited state are proposed to be populated in the experiment. The similar observation has been made for $\mathrm{Cr}_{2} \mathrm{O}_{2}{ }^{-/ 0} \cdot 39$

The IR spectrum of $\mathrm{Cr}_{3} \mathrm{O}^{+} \cdot \mathrm{He}_{2}$ has a rather low signal-to-noise ratio, but shows a pronounced peak at $700 \mathrm{~cm}^{-1}$ (see Figure 2c). The lowest state is found to be ${ }^{6} \mathrm{~A}^{\prime}$ for a propeller-like isomer within the $\mathrm{C}_{s}$ symmetry. $\mathrm{A}_{3 h}$ isomer with a high spin ${ }^{16} \mathrm{~A}^{\prime}$ electronic state is identified as the first excited state. The predicted IR spectrum of the ${ }^{6} \mathrm{~A}^{\prime}$ ground state is in agreement with the experimental IRPD spectrum, i.e. the IR-active peak observed at $700 \mathrm{~cm}^{-1}$ is reproduced at $707 \mathrm{~cm}^{-1}$. The IR spectrum of the first excited state does not match the experimental spectrum, because its intense mode is about $150 \mathrm{~cm}^{-1}$ redshifted from the experimental feature.
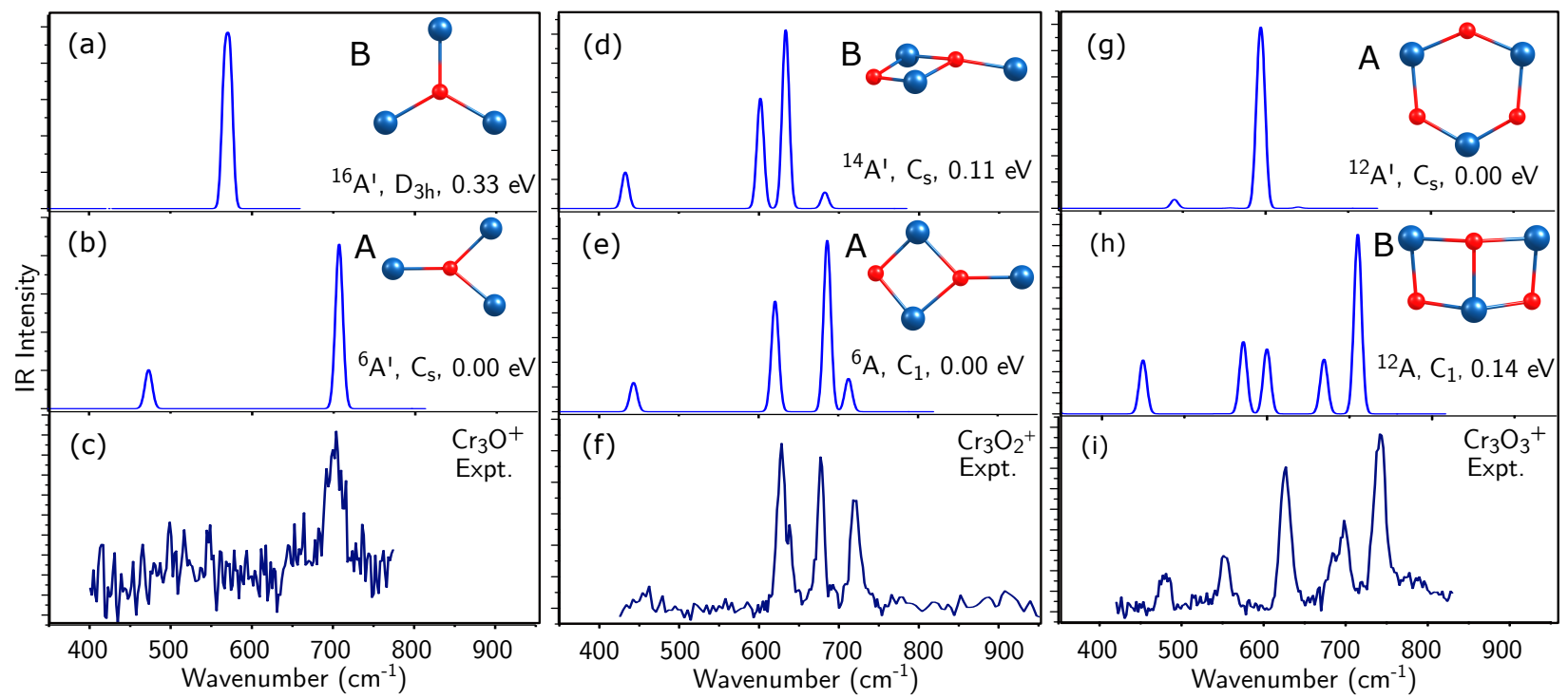

Figure 2: Experimental IRPD spectra and simulated harmonic IR spectra of $\mathrm{Cr}_{3} \mathrm{O}^{+}, \mathrm{Cr}_{3} \mathrm{O}_{2}{ }^{+}$, and $\mathrm{Cr}_{3} \mathrm{O}_{3}{ }^{+}$. The geometries of lowest-lying states for each cluster are shown as insets.

The IRPD spectrum of $\mathrm{Cr}_{3} \mathrm{O}_{2}{ }^{+}$, measured on the $\mathrm{Cr}_{3} \mathrm{O}_{2}{ }^{+} \cdot \mathrm{He}$ complex, has three clear bands at 630,680 , and $725 \mathrm{~cm}^{-1}$. The simulated IR spectra of the sextet ground and a 14-et first excited state (see Figure 2d and 2e) were used to determine the experimentally populated structure of $\mathrm{Cr}_{3} \mathrm{O}_{2}{ }^{+}$. For the ${ }^{6} \mathrm{~A}$ state state, the TPSS method reproduces the three main experimental infrared active vibrations. Simulated spectrum of this isomers using other DFT functionals are similar and can be found in the SI (Figure S11). We note that calculated 
harmonic IR spectra are typically reliable to reproduce the number and the frequencies of the vibrational modes that are seen experimental, but are less accurate for predicting the relative intensities of those modes. The main reasons here for are the multiple photon aspect and the statistical dissociation of the cluster-rare gas complexes in the experiment, which are not included in the computations. A detailed discussion of the consequences of those effects can be found in ref. 40. So we conclude that the sextet state is likely the isomer that is observed in the experiment. However, the first excited state ${ }^{14} \mathrm{~A}^{\prime}$ is quite close to the ground state in terms of energy $(+0.11 \mathrm{eV}$ at the TPSS level), and its simulated spectrum is similar to the one of the ${ }^{6} \mathrm{~A}$ state. Therefore, both isomers may coexist in the experiment.

For $\mathrm{Cr}_{3} \mathrm{O}_{3}{ }^{+}$two isomers with a high spin 12-tet (noted as A and B in Figures $2 \mathrm{~g}$ and $2 \mathrm{~h}$, respectively) are energetically competitive. Although the calculated relative energy of isomer $\mathrm{B}(+0.14 \mathrm{eV})$ is slightly higher than that of isomer A, its simulated IR spectrum agrees much better with the experiment performed on the $\mathrm{Cr}_{3} \mathrm{O}_{3}{ }^{+} \cdot \mathrm{Ne}$ complex with regard to the frequency of the modes and the number of peaks. We, therefore, assign the spectrum to isomer B. Note that the relative energy difference is comparable to the accuracy of the computational method. As reported in Table S6 of the supporting information, different hybrid and metaGGA functionals either predict isomer A (TPSS, B3LYP, B3PW91, TPSSH) or isomer B (M06L, B3P86) as most stable isomer with a maximal energy difference of 0.17 $\mathrm{eV}$.

The IRPD spectrum of $\mathrm{Cr}_{4} \mathrm{O}_{4}{ }^{+}$(see Figure 3) shows several vibrational bands, which are grouped in three ranges: $550-600 \mathrm{~cm}^{-1}, 650-710 \mathrm{~cm}^{-1}$ and $750-790 \mathrm{~cm}^{-1}$. In the computational study, many different local minima were obtained for $\mathrm{Cr}_{4} \mathrm{O}_{4}{ }^{+}$. Two structural motifs were found to be more stable: a cage-like structure (isomer A) and a ring-like structure (isomer B). Two most stable isomers of $\mathrm{Cr}_{4} \mathrm{O}_{4}{ }^{+}$, their lowest energy electronic states, and corresponding simulated IR spectra are visualized in Figure 3. The simulated IR spectrum of isomer $\mathrm{A}$ in the octet state is in substantially better agreement with the experiment performed on the $\mathrm{Cr}_{4} \mathrm{O}_{4}{ }^{+} \cdot \mathrm{Ne}$ complex, than that of isomer B. The only discrepancy is a redshift 
of the simulated spectrum by $40 \mathrm{~cm}^{-1}$, particularly for the intense bands in the $670-760 \mathrm{~cm}^{-1}$ range. However, with the B3LYP, B3PW91 and B3P86 functional (see Figure S13 in the SI), the redshift for these bands is much smaller $\left(<10 \mathrm{~cm}^{-1}\right)$. The IR spectrum of the cyclic isomer B only predicts two IR-active bands and cannot explain the more complex spectrum observed in the experiment.

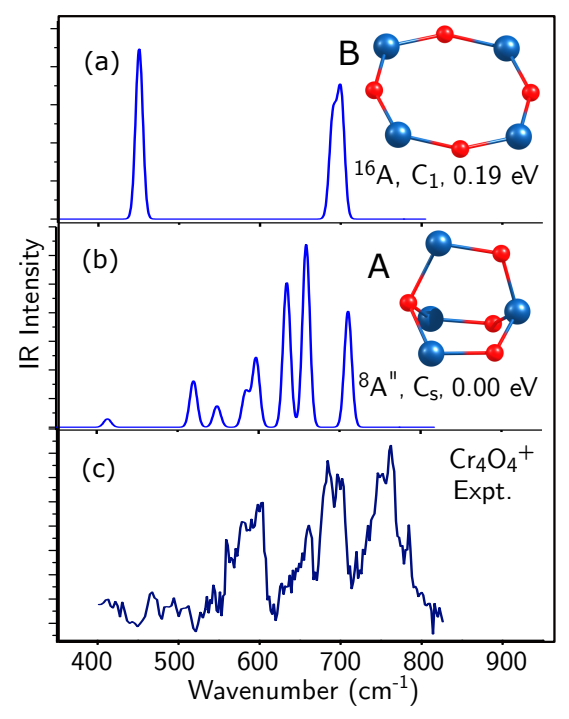

Figure 3: Experimental IRPD spectra and simulated harmonic IR spectra of $\mathrm{Cr}_{4} \mathrm{O}_{4}{ }^{+}$. The geometries of two most stable isomers are shown as insets.

From the above results, one can recognize that different structural motifs result in different IR spectra. In certain cases also similar structural motifs with a different spin state and thus different magnetic configurations can be distinguished by their IR spectra. This is the case for $\mathrm{Cr}_{4} \mathrm{O}_{4}{ }^{+}$. Figure 4 presents four different spin configurations (quartet, sextet, octet, and dectet) of structural isomer $\mathrm{A}$ of $\mathrm{Cr}_{4} \mathrm{O}_{4}{ }^{+}$, with the octet spin state having the lowest energy. The simulated IR spectra of those different spin states are distinct from each other. This implies that the IRPD technique is, in this specific case, a suitable tool to not only probe the geometric structure but also spin configurations. This is a clear evidence demonstrating direct relation between the IRPD technique and magnetic properties. 


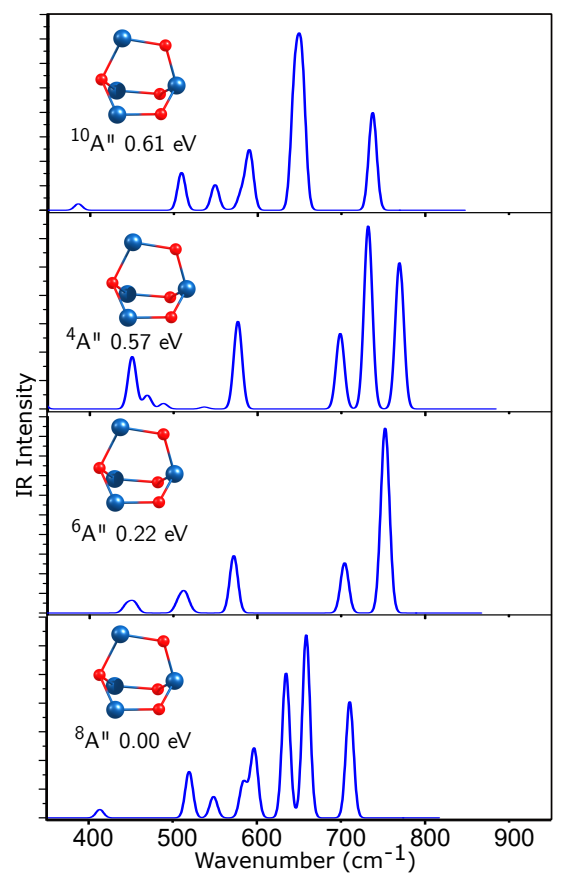

Figure 4: Simulated harmonic IR spectra of structural isomer $\mathrm{A}$ of $\mathrm{Cr}_{4} \mathrm{O}_{4}^{+}$in four different spin states.

\section{Magnetic Properties}

After assigning the geometric structures and the electronic states of the chromium oxide clusters by comparing the measured with simulated IR spectra, computations can be used to analyze the magnetic properties of the clusters. Local spin moments of individual atoms, which combine to give total spin magnetic moments of clusters, are graphically presented in Figure 5. Values of these local spin magnetic moments are given in Table 1. Also $\mathrm{Cr}_{3}{ }^{+}$, $\mathrm{Cr}_{3} \mathrm{O}_{4}{ }^{+}$and $\mathrm{Cr}_{3} \mathrm{O}_{5}{ }^{+}$are added to the figure and the table. For these sizes we have only computational results and no assignment of their structure based on the infrared spectra was performed. The spin magnetic configurations of the clusters can be divided into two groups: i) clusters in which chromium spin moments are ferromagnetically coupled $\left(\mathrm{Cr}_{2} \mathrm{O}_{2}{ }^{+}, \mathrm{Cr}_{3} \mathrm{O}_{3}{ }^{+}\right.$, $\mathrm{Cr}_{3} \mathrm{O}_{4}{ }^{+}$, and $\mathrm{Cr}_{3} \mathrm{O}_{5}^{+}$) and ii) clusters in which metallic spin moments are ferrimagnetically coupled $\left(\mathrm{Cr}_{3}{ }^{+}, \mathrm{Cr}_{3} \mathrm{O}^{+}, \mathrm{Cr}_{3} \mathrm{O}_{2}{ }^{+}\right.$, and $\left.\mathrm{Cr}_{4} \mathrm{O}_{4}{ }^{+}\right)$. In the ground-state structures of $\mathrm{Cr}_{2} \mathrm{O}_{2}{ }^{+}$, $\mathrm{Cr}_{3} \mathrm{O}_{3}{ }^{+}, \mathrm{Cr}_{3} \mathrm{O}_{4}{ }^{+}$, and $\mathrm{Cr}_{3} \mathrm{O}_{5}{ }^{+}$all spin magnetic moments of the chromium atoms are parallel, which leads to high values of $7,11,9$, and $7 \mu_{B}$, respectively. $\mathrm{In}_{\mathrm{Cr}_{3}}{ }^{+}, \mathrm{Cr}_{3} \mathrm{O}^{+}, \mathrm{Cr}_{3} \mathrm{O}_{2}{ }^{+}$and 
$\mathrm{Cr}_{4} \mathrm{O}_{4}{ }^{+}$the total spin magnetic moment is reduced compared to the maximal values, resulting in spin moments of $3,5,5$ and $7 \mu_{B}$, respectively.

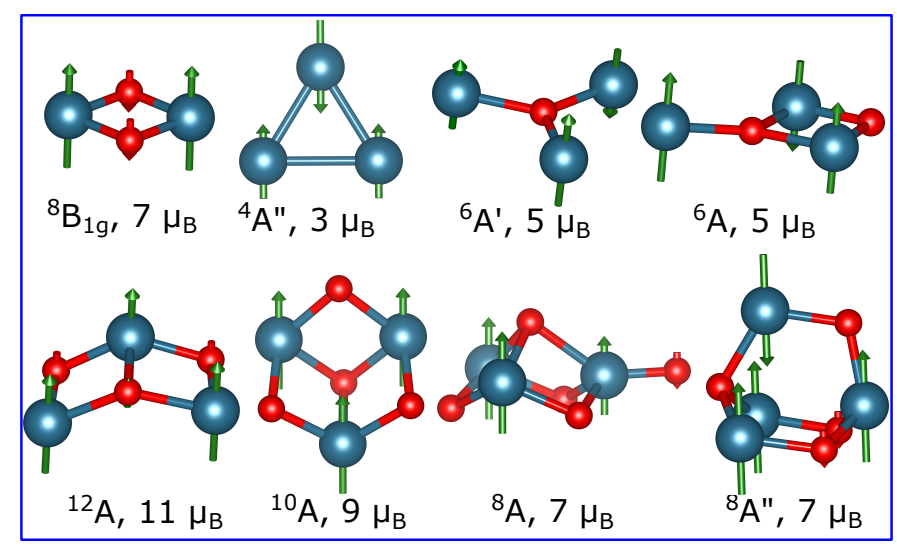

Figure 5: Structures of $\mathrm{Cr}_{2} \mathrm{O}_{2}^{+}, \mathrm{Cr}_{3} \mathrm{O}^{+}, \mathrm{Cr}_{3} \mathrm{O}_{2}{ }^{+}, \mathrm{Cr}_{3} \mathrm{O}_{3}{ }^{+}$, and $\mathrm{Cr}_{4} \mathrm{O}_{4}{ }^{+}$as assigned by comparison of simulated IR spectra with the experimental IRPD spectra. In addition, the structures of $\mathrm{Cr}_{3}{ }^{+}, \mathrm{Cr}_{3} \mathrm{O}_{4}{ }^{+}$and $\mathrm{Cr}_{3} \mathrm{O}_{5}{ }^{+}$, for which no IRPD data is available, are added to the figure. Spin magnetic moments of $\mathrm{Cr}_{3}{ }^{+}, \mathrm{Cr}_{3} \mathrm{O}_{4}{ }^{+}$and $\mathrm{Cr}_{3} \mathrm{O}_{5}{ }^{+}$were calculated from their energetically lowest geometry and electronic states. Chromium and oxygen atoms are represented by blue and red balls, respectively. Green (red) arrows indicate the orientation of the local spin magnetic moments $\left(>0.05 \mu_{B}\right)$ on the chromium (oxygen) atoms. 


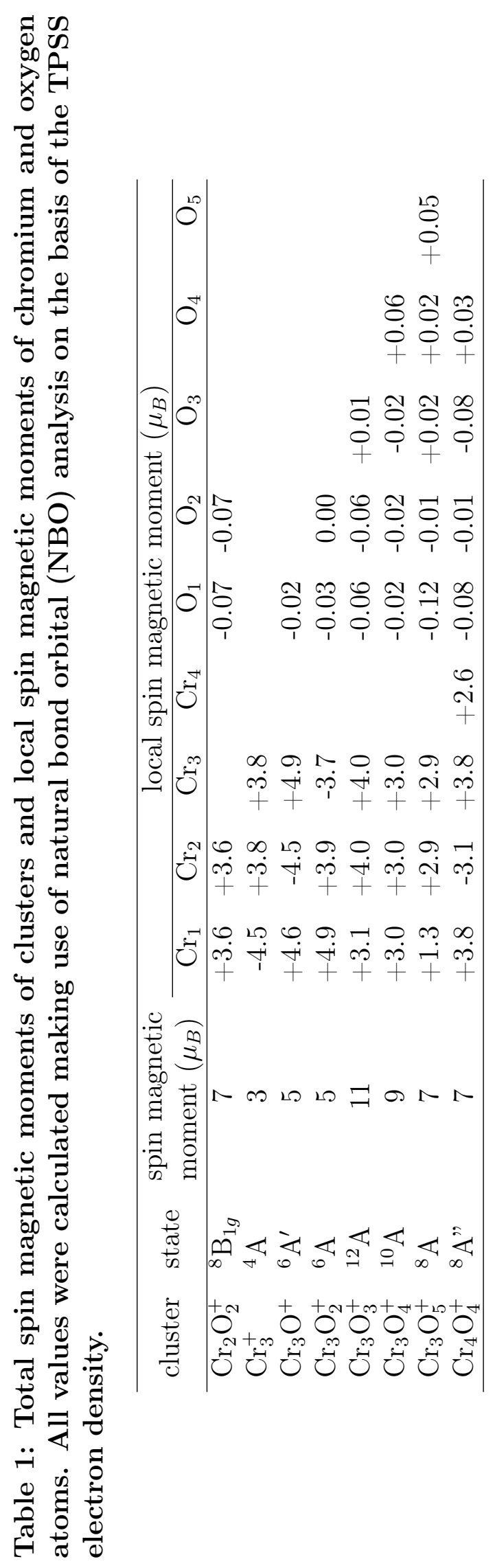


$3 d-3 d$ bonding-formation and superexchange-type interactions were demonstrated to underlie the corresponding magnetic properties in pure chromium clusters $\left(\mathrm{Cr}_{2}{ }^{0}, \mathrm{Cr}_{3}{ }^{+/ 0}\right)^{2,41,42}$ and dichromium oxide clusters $\left(\mathrm{Cr}_{2} \mathrm{O}_{2}{ }^{-/ 0}\right.$, and $\left.\mathrm{Cr}_{2} \mathrm{O}_{3}{ }^{-/ 0}\right) \cdot{ }^{8,9,43}$ In this work, we find small antiferromagnetic spin moments on the oxygen atoms in $\mathrm{Cr}_{2} \mathrm{O}_{2}{ }^{+}$and $\mathrm{Cr}_{3} \mathrm{O}_{3}{ }^{+}$. Coupling between the chromium and oxygen sites in these clusters occurs through the hybridization between $\mathrm{Cr} 3 d$ and $\mathrm{O} 2 p$ orbitals. Such an orbital interaction is known as superexchange and enhances the parallel spin coupling between chromium atoms. ${ }^{8,9,43}$ Figure 6 presents the total density of states (TDOS) and partial density of states (PDOS) of $\mathrm{Cr}_{3} \mathrm{O}_{3}{ }^{+}$and $\mathrm{Cr}_{3} \mathrm{O}^{+}$. In the case of $\mathrm{Cr}_{3} \mathrm{O}_{3}{ }^{+}$, the PDOS (Figure 6c) reveals, particularly for the alpha occupied orbitals, strong hybridization between the chromium $3 d$ and the oxygen $2 p$ orbitals in the -15.0 to $-9.0 \mathrm{eV}$ range (-9.0 eV is the energy of the HOMO). $\mathrm{The} \mathrm{Cr}_{3}{ }^{+}, \mathrm{Cr}_{3} \mathrm{O}^{+}, \mathrm{Cr}_{3} \mathrm{O}_{2}{ }^{+}$, and $\mathrm{Cr}_{4} \mathrm{O}_{4}{ }^{+}$clusters disclose ferrimagnetic magnetic behavior, in which one of chromium atoms has its magnetic moment in the reverse direction of the others. By analyzing interaction between the two closest chromium atoms in the $\mathrm{C}_{\mathrm{s}}$ isomer of $\mathrm{Cr}_{3} \mathrm{O}^{+}$, bonding-like features are found between those two chromium atoms (see Figure S14). The 3d PDOS of the antiferromagnetically coupled chromium atoms of this cluster show energetic overlap between alpha and beta $3 d$ occupied orbitals in the -10 to $-8.5 \mathrm{eV}$ range (Figure $6 \mathrm{~d}$ ). Therefore, $3 d-$ $3 d$-like bonding between two closest chromium atoms causes an antiferromagnetic coupling of the local spin magnetic moments, similar as in the $\mathrm{Cr}_{2}$ dimers. ${ }^{41,42}$ Similar bonding-type formation is believed to be present in $\mathrm{Cr}_{3} \mathrm{O}_{2}{ }^{+}$and $\mathrm{Cr}_{4} \mathrm{O}_{4}{ }^{+}$(see Figures S14 and S15), where the spin moment of one of the chromium atoms in antiparallel to the other ones. 

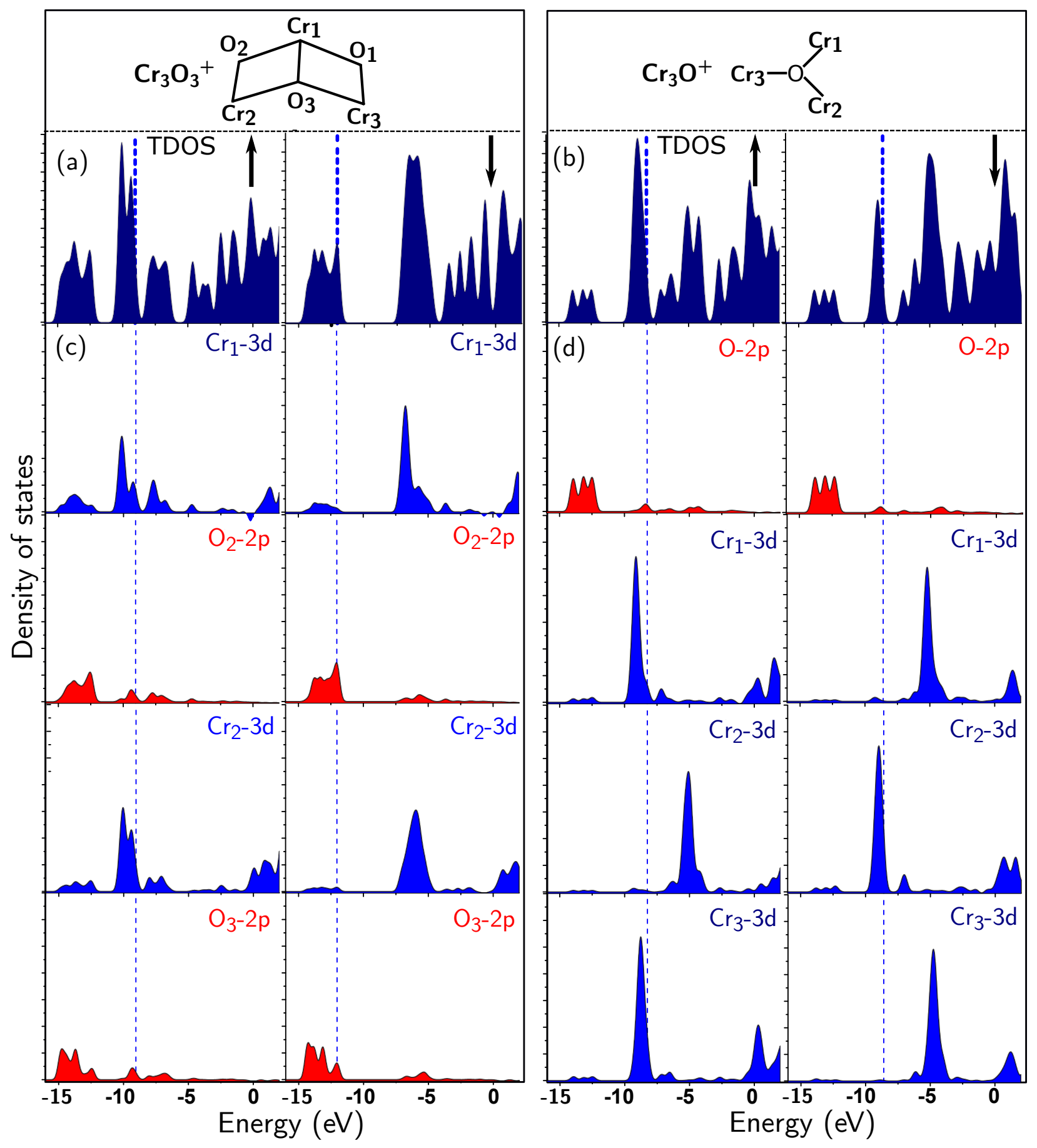

Figure 6: Total density of states (TDOS) and partial density of states (PDOS) of $\mathrm{Cr}_{3} \mathrm{O}_{3}{ }^{+}$ (left part) and $\mathrm{Cr}_{3} \mathrm{O}^{+}$(right part). For each part the left (right) side corresponds to alpha or up (beta or down) orbitals as indicated by the black arrows. (a) TDOS of $\mathrm{Cr}_{3} \mathrm{O}_{3}{ }^{+}$, (b) TDOS of $\mathrm{Cr}_{3} \mathrm{O}^{+}$, (c) PDOS of $\mathrm{Cr}_{3} \mathrm{O}_{3}{ }^{+}$, and (d) PDOS of $\mathrm{Cr}_{3} \mathrm{O}^{+}$. For PDOS, the projections in oxygen-2p (chromium-3d) orbitals are shown in red (blue). Atoms are numbered according to the structures shown in the insets. Vertical short dashed lines indicate the HOMO. 
Next, we investigate the effect of atom-by-atom oxygen addition to the geometry and magnetism of the $\mathrm{Cr}_{3}{ }^{+}$metallic cluster. Figure 7 presents the magnetic evolution of $\mathrm{Cr}_{3} \mathrm{O}_{\mathrm{n}}{ }^{+}$ $(\mathrm{n}=0-5)$. With the addition of oxygen atoms, the total magnetic moment increases from $\mathrm{n}=0\left(3 \mu_{B}\right)$ to $\mathrm{n}=3\left(11 \mu_{B}\right)$. Such a strong increase of the total magnetic moment implies that direct $3 d-3 d$ bonding-type interaction, which favors antiferromagnetic coupling, becomes weaker upon addition of oxygen atoms, reducing the $3 d-3 d$ interaction. The $3 d-3 d$ bonding-type formation is dominated by the superexchange interaction in $\mathrm{Cr}_{3} \mathrm{O}_{3}{ }^{+}$, leading to a high total spin magnetic moment $\left(11 \mu_{B}\right)$. The total spin magnetic moment is gradually reduced if more oxygen atoms are added $(n=4$ and $n=5)$. Finally the magnetic moment is expected to be quenched as was computationally found for $\mathrm{Cr}_{2} \mathrm{O}_{\mathrm{n}}{ }^{11}(\mathrm{n} \geq 6)$. This result unravels how the oxygen concentration affects and controls the total magnetic properties of trichromium oxides.

Overall the evolution of the structures and the magnetic configurations with the oxygen concentration indicates that the highest magnetic moments, i.e. parallel alignment of the local $\mathrm{Cr}$ spin magnetic moments, are obtained in suboxides that have enough oxygen atoms to form $\mathrm{Cr}-\mathrm{O}-\mathrm{Cr}$ bridges with a unique $\mathrm{O}$ atom between each pair of $\mathrm{Cr}$ atoms. For $\mathrm{Cr}_{3} \mathrm{O}_{\mathrm{n}}{ }^{+}$this occurs in $\mathrm{Cr}_{3} \mathrm{O}_{3}{ }^{+}$. In this cluster the superexchange interaction is maximized and occurs without strong reduction of the local magnetic moments on the involved $\mathrm{Cr}$ atom (see Table 1). Addition of more oxygen atoms (i.e. in $\mathrm{Cr}_{3} \mathrm{O}_{4}{ }^{+}$and $\mathrm{Cr}_{3} \mathrm{O}_{5}{ }^{+}$) results in the formation of $\mathrm{Cr}-\mathrm{O}$ bonds with individual $\mathrm{Cr}$ atoms and the delocalization of the $\mathrm{Cr} 3 d$ electrons in $\mathrm{Cr}-\mathrm{O}$ shared orbitals. Such capturing of unpaired $\mathrm{Cr} 3 \mathrm{~d}$ electrons reduces the Cr local magnetic moments. The observation of this magnetic configuration dependence on the oxygen concentration is empirical. An in-debt explanation may be the subject of follow-up studies. 


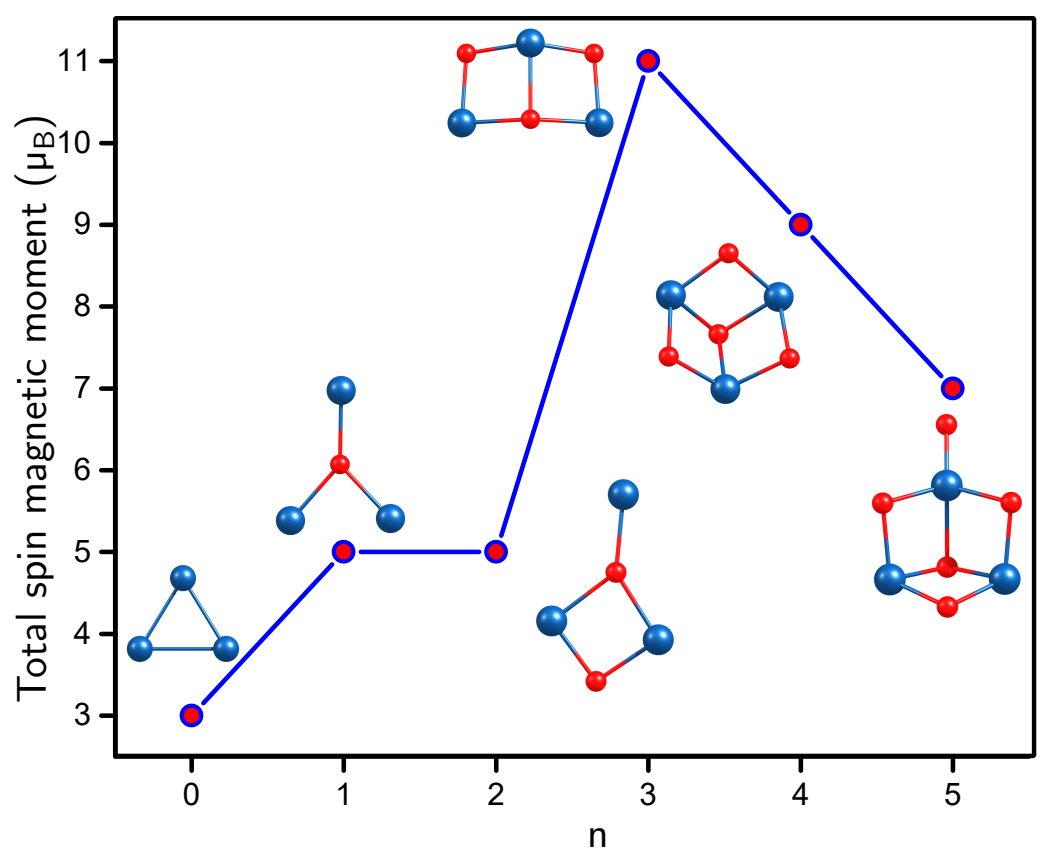

Figure 7: Evolution of the total spin magnetic moment for $\mathrm{Cr}_{3} \mathrm{O}_{\mathrm{n}}{ }^{+}(\mathrm{n}=0-5)$ clusters.

\section{Conclusion}

In conclusion, several cationic chromium-rich oxides were synthesized and characterized through IR spectral characteristics. The magnetic states of these clusters were studied to reveal insights into their magnetic properties. Oxygen plays an important role in controlling the magnetic configurations. Superexchange interaction through oxygen bridging sites causes ferromagnetic coupling of the chromium atoms in $\mathrm{Cr}_{2} \mathrm{O}_{2}{ }^{+}$and $\mathrm{Cr}_{3} \mathrm{O}_{3}{ }^{+}$. The ferrimagnetic behavior of $\mathrm{Cr}_{3} \mathrm{O}^{+}, \mathrm{Cr}_{3} \mathrm{O}_{2}{ }^{+}$, and $\mathrm{Cr}_{4} \mathrm{O}_{4}{ }^{+}$is attributed to the bonds between the metal atoms, in which $3 d$ orbitals of nearby chromium atoms directly interact with each other resulting in lower total spin magnetic moments. This study indicates that the highest possible total magnetic moments are obtained in suboxides with a comparable number of oxygen and chromium atoms that have a unique oxygen atom in a single $\mathrm{Cr}-\mathrm{O}-\mathrm{Cr}$ bridge between each pair of $\mathrm{Cr}$ atoms. The addition of more oxygen atoms enhances the delocalization of the $\mathrm{Cr}$ $3 d$ electrons and reduces the magnetic moment. 


\section{Acknowledgement}

This research has been supported by the KU Leuven Research Council (GOA progam) and by the Research Foundation - Flanders (FWO project G.0B41.15N). The authors are grateful to Dr. Liviu Ungur for his kind assistance with the MOLCAS program. The authors acknowledge support of the Stichting voor Fundamenteel Onderzoek der Materie (FOM) in providing beam time on FELIX and highly appreciate the skillful assistance of the FELIX staff.

\section{Supporting Information Available}

Supporting Information Available: Computational details for $\mathrm{Cr}_{2} \mathrm{O}_{2}{ }^{+}$; geometrical structures and relative energies of different electronic states of $\mathrm{Cr}_{2} \mathrm{O}_{2}{ }^{+}$; computational assessment of the influence of the rare gas messenger atoms on the IR spectra; structures of the lowest energy isomers of $\mathrm{Cr}_{3} \mathrm{Om}^{+}(m=1-5)$ and $\mathrm{Cr}_{4} \mathrm{O}_{4}^{+}$; calculated relative energies of $\mathrm{Cr}_{3} \mathrm{Om}^{+}$ $(m=1-5)$ and $\mathrm{Cr}_{4} \mathrm{O}_{4}{ }^{+}$at different DFT levels; comparison of the IRPD spectra with simulated spectra at different DFT levels; AdNDP analysis of possible two-center bonding; and partial density of states of $\mathrm{Cr}_{2} \mathrm{O}_{2}{ }^{+}, \mathrm{Cr}_{3} \mathrm{O}_{2}{ }^{+}$and $\mathrm{Cr}_{4} \mathrm{O}_{4}{ }^{+}$.

\section{References}

(1) Fawcett, E. Spin-density-wave antiferromagnetism in chromium. Rev. Mod. Phys. 1988, 60, 209-283.

(2) Bondybey, V.; English, J. Electronic structure and vibrational frequency of $\mathrm{Cr}_{2}$. Chem. Phys. Lett. 1983, 94, 443-447.

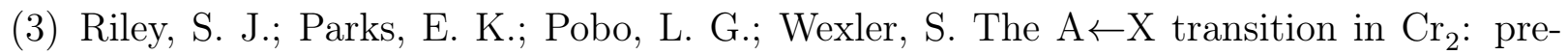


dissociation, isotope effects, and the 1-1 sequence band. J. Chem. Phys. 1983, 79, $2577-2582$.

(4) Zamudio-Bayer, V.; Hirsch, K.; Langenberg, A.; Niemeyer, M.; Vogel, M.; Ławicki, A.; Terasaki, A.; Lau, J. T.; von Issendorff, B. Maximum spin polarization in chromium dimer cations as demonstrated by X-ray magnetic circular dichroism spectroscopy. Angew. Chem. Int. Ed. 2015, 54, 4498-4501.

(5) Janssens, E.; Hou, X. J.; Neukermans, S.; Wang, X.; Silverans, R. E.; Lievens, P.; Nguyen, M. T. The exchange coupling in $\mathrm{Cr}_{3} \mathrm{O}_{\mathrm{n}}(\mathrm{n}=0-3)$ clusters. J. Phys. Chem. A 2007, 111, 4150-4157.

(6) Cheng, H.; Wang, L.-S. Dimer growth, structural transition, and antiferromagnetic ordering of small chromium clusters. Phys. Rev. Lett. 1996, r7, 51-54.

(7) López-Estrada, O.; López-Olay, S.; Aburto, A.; Orgaz, E. Unexpected high spin polarization in $\mathrm{Cr}_{4}$ cluster. J. Phys. Chem. C 2016, 120, 23892-23897.

(8) Tono, K.; Terasaki, A.; Ohta, T.; Kondow, T. Chemical control of magnetism: oxidation-induced ferromagnetic spin coupling in the chromium dimer evidenced by photoelectron spectroscopy. Phys. Rev. Lett. 2003, 90, 133402.

(9) Tono, K.; Terasaki, A.; Ohta, T.; Kondow, T. Chemically induced ferromagnetic spin coupling: electronic and geometric structures of chromium-oxide cluster anions, $\mathrm{Cr}_{2} \mathrm{O}_{\mathrm{n}}{ }^{-}$ ( $\mathrm{n}=1-3)$, studied by Photoelectron spectroscopy. J. Chem. Phys. 2003, 119, 1122111227.

(10) Reddy, B. V.; Khanna, S. N.; Ashman, C. Oscillatory magnetic coupling in $\mathrm{Cr}_{2} \mathrm{O}_{\mathrm{n}}$ (n $=1-6)$ clusters. Phys. Rev. B 2000, 61, 5797-5801.

(11) Gutsev, G. L.; Bozhenko, K. V.; Gutsev, L. G.; Utenyshev, A. N.; Aldoshin, S. M. 
Transitions from stable to metastable states in the $\mathrm{Cr}_{2} \mathrm{O}_{\mathrm{n}}$ and $\mathrm{Cr}_{2} \mathrm{O}_{\mathrm{n}}{ }^{-}$series, $\mathrm{n}=1-$ 14. J. Phys. Chem. A 2017, 121, 845-854, PMID: 28059512.

(12) Li, S.; Zhai, H.-J.; Wang, L.-S.; Dixon, D. A. Structural and Electronic properties of reduced transition metal oxide clusters, $\mathrm{M}_{4} \mathrm{O}_{10}$ and $\mathrm{M}_{4} \mathrm{O}_{10}{ }^{-}(\mathrm{M}=\mathrm{Cr}, \mathrm{W})$, from photoelectron spectroscopy and quantum chemical calculations. J. Phys. Chem. A 2012, 116, 5256-5271, PMID: 22551114.

(13) Zhai, H.-J.; Li, S.; Dixon, D. A.; Wang, L.-S. Probing the electronic and structural properties of chromium oxide clusters $\left(\mathrm{CrO}_{3}\right)_{\mathrm{n}}{ }^{-}$and $\left(\mathrm{CrO}_{3}\right)_{\mathrm{n}}(\mathrm{n}=1-5)$ : photoelectron spectroscopy and Density Functional Calculations. J. Am. Chem. Soc. 2008, 130, 51675177, PMID: 18327905.

(14) Pakiari, A. H.; Shariati, S. Geometry and electronic structure of ultrafine/nanoparticle chromium clusters $\left(\mathrm{Cr}_{\mathrm{n}}, \mathrm{n}=2-5\right)$ and their interaction with oxygen (Triplet) and ethylene molecules: a DFT-NBO study. Comput. Theor. Chem. 2016, 1084, 169-178.

(15) Shah, E. V.; Roy, D. R. Magnetic switching in $\mathrm{Cr}_{\mathrm{x}}(\mathrm{x}=2-8)$ and its oxide cluster series. J. Magn. Magn. Mat. 2018, 451, 32-37.

(16) Ding, X.-L.; Xue, W.; Ma, Y.-P.; Wang, Z.-C.; He, S.-G. Density functional study on cage and noncage $\left(\mathrm{Fe}_{2} \mathrm{O}_{3}\right)_{\mathrm{n}}$ clusters. J. Chem. Phys. 2009, 130, 014303.

(17) Kirilyuk, A.; Fielicke, A.; Demyk, K.; von Helden, G.; Meijer, G.; Rasing, T. Ferrimagnetic cagelike $\mathrm{Fe}_{4} \mathrm{O}_{6}$ cluster: structure determination from infrared dissociation spectroscopy. Phys. Rev. B 2010, 82, 020405.

(18) Logemann, R.; demWijs, s. A.; Katsnelson, M. I.; Kirilyuk, A. Geometric, electronic, and magnetic structure of $\mathrm{Fe}_{\mathrm{x}} \mathrm{O}_{\mathrm{y}}{ }^{+}$clusters. Phys. Rev. B 2015, 92, 144427.

(19) Erlebach, A.; Huhn, C.; Jana, R.; Sierka, M. Structure and magnetic properties of $\left(\mathrm{Fe}_{2} \mathrm{O}_{3}\right)_{\mathrm{n}}$ clusters $(\mathrm{n}=1-5)$. Phys. Chem. Chem. Phys. 2014, 16, 26421-26426. 
(20) Janssens, E.; Santambrogio, G.; Brümmer, M.; Wöste, L.; Lievens, P.; Sauer, J.; Meijer, G.; Asmis, K. R. Isomorphous substitution in bimetallic oxide clusters. Phys. Rev. Lett. 2006, 96, 233401.

(21) Santambrogio, G.; Janssens, E.; Li, S.; Siebert, T.; Meijer, G.; Asmis, K. R.; Döbler, J.; Sierka, M.; Sauer, J. Identification of conical structures in small aluminum oxide clusters: infrared spectroscopy of $\left(\mathrm{Al}_{2} \mathrm{O}_{3}\right)_{1-4}(\mathrm{AlO})^{+}$. J. Am. Chem. Soc. 2008, 130, 1514315149.

(22) Brümmer, M.; Kaposta, C.; Santambrogio, G.; Asmis, K. R. Formation and photodepletion of cluster ion-messenger atom complexes in a cold ion trap: infrared spectroscopy of $\mathrm{VO}^{+}, \mathrm{VO}_{2}^{+}$, and $\mathrm{VO}_{3}^{+}$. J. Chem. Phys. 2003, 119, 12700-12703.

(23) Asmis, K. R.; Sauer, J. Mass-selective vibrational spectroscopy of vanadium oxide cluster ions. Mass Spectrom. Rev. 2007, 26, 542-562.

(24) van Dijk, C. N.; Roy, D. R.; Fielicke, A.; Rasing, T.; Reber, A. C.; Khanna, S. N.; Kirilyuk, A. Structure investigation of $\mathrm{Co}_{\mathrm{x}} \mathrm{O}_{\mathrm{y}}{ }^{+}(\mathrm{x}=3-6, \mathrm{y}=3-8)$ clusters by IR vibrational spectroscopy and DFT calculations. Eur. Phys. J. D 2014, 68, 357.

(25) Goebbert, D. J.; Meijer, G.; Asmis, K. R. 10 K ring electrode trap-tandem mass spectrometer for infrared spectroscopy of mass selected ions. AIP Conference Proceedings 2009, 1104, 22-29.

(26) Oepts, D.; Van der Meer, A.; Van Amersfoort, P. The free-electron-laser user facility FELIX. Infrared Phys. Techn. 1995, 36, 297-308.

(27) Ferrari, P.; Vanbuel, J.; Li, Y.; Liao, T.-W.; Janssens, E.; Lievens, P. In Gas-phase synthesis of nanoparticles; Huttel, Y., Ed.; Wiley-VCH Verlag GmbH \& Co. KGaA, pp 59-78. 
(28) Wang, Y.; Lv, J.; Zhu, L.; Ma, Y. Crystal structure prediction via particle-swarm optimization. Phys. Rev. B 2010, 82, 094116.

(29) Balabanov, N. B.; Peterson, K. A. Systematically convergent basis sets for transition metals. I. all-electron correlation consistent basis sets for the 3d elements Sc-Zn. J. Chem. Phys. 2005, 123, 064107.

(30) Dunning, T. H., Jr. Gaussian basis sets for use in correlated molecular calculations. I. the atoms boron through neon and hydrogen. J. Chem. Phys. 1989, 90, 1007-1023.

(31) Lee, C.; Yang, W.; Parr, R. G. Development of the colle-salvetti correlation-energy formula into a functional of the electron density. Phys. Rev. B 1988, 37, 785-789.

(32) Becke, A. D. Density-functional exchange-energy approximation with correct asymptotic behavior. Phys. Rev. A 1988, 38, 3098-3100.

(33) Becke, A. D. Density-functional thermochemistry. III. the role of exact exchange. $J$. Chem. Phys. 1993, 98, 5648-5652.

(34) Perdew, J. P. Density-functional approximation for the correlation energy of the inhomogeneous electron gas. Phys. Rev. B 1986, 33, 8822-8824.

(35) Tao, J.; Perdew, J. P.; Staroverov, V. N.; Scuseria, G. E. Climbing the density functional ladder: nonempirical meta-generalized gradient approximation designed for molecules and solids. Phys. Rev. Lett. 2003, 91, 146401.

(36) Zhao, Y.; Truhlar, D. G. A new local density functional for main-group thermochemistry, transition metal bonding, thermochemical kinetics, and noncovalent interactions. J. Chem. Phys. 2006, 125, 194101.

(37) Frisch, M. J.; Trucks, G. W.; Schlegel, H. B.; Scuseria, G. E.; Robb, M. A.; Cheeseman, J. R.; Scalmani, G.; Barone, V.; Mennucci, B.; Petersson, G. A. et al. Gaussian-09 Revision E.01. Gaussian Inc. Wallingford CT 2009. 
(38) Grimme, S.; Antony, J.; Ehrlich, S.; Krieg, H. A consistent and accurate ab initio parametrization of density functional dispersion correction (DFT-D) for the 94 elements H-Pu. J. Chem. Phys. 2010, 132, 154104.

(39) Pham, L. N.; Nguyen, M. T. Another look at photoelectron spectra of the anion $\mathrm{Cr}_{2} \mathrm{O}_{2}{ }^{-}$: multireference characters and energetic degeneracy. J. Chem. Theory Comput. 2018, $14,4833-4843$.

(40) Calvo, F.; Li, Y.; Kiawi, D. M.; Bakker, J. M.; Parneix, P.; Janssens, E. Nonlinear effects in infrared action spectroscopy of silicon and vanadium oxide clusters: experiment and kinetic modeling. Phys. Chem. Chem. Phys. 2015, 17, 25956-25967.

(41) Vancoillie, S.; Malmqvist, P. A.; Veryazov, V. Potential energy surface of the chromium dimer re-re-revisited with multiconfigurational perturbation theory. J. Chem. Theory Comput. 2016, 12, 1647-1655.

(42) Simard, B.; Lebeault-Dorget, M.-A.; Marijnissen, A.; ter Meulen, J. J. Photoionization spectroscopy of dichromium and dimolybdenum: ionization potentials and bond energies. J. Chem. Phys. 1998, 108, 9668-9674.

(43) Paul, S.; Misra, A. Magnetic properties of $\mathrm{Cr}_{2} \mathrm{O}_{\mathrm{n}}{ }^{-}$clusters: a theoretical study. J. Mol. Struc.: THEOCHEM 2009, 895, 156-160. 
Graphical TOC Entry

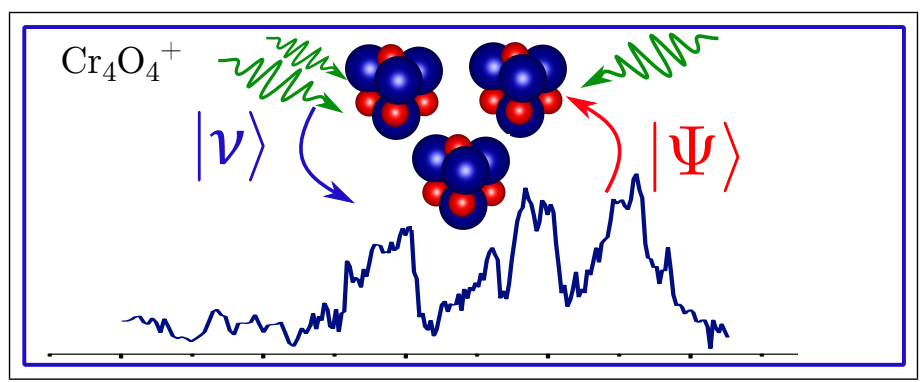

